\title{
Understanding Dissolution and Crystallization with Imaging: A Surface Point of View
}

\author{
Dunja Novakovic, ${ }^{*}, \dagger$ Antti Isomäki, ${ }^{\ddagger}$ Bibi Pleunis, ${ }^{\dagger}$ Sara J. Fraser-Miller, ${ }^{\perp}$ Leena Peltonen, ${ }^{\dagger}$
} Timo Laaksonen," and Clare J. Strachan ${ }^{\dagger}$

${ }^{\dagger}$ Drug Research Program, Division of Pharmaceutical Chemistry and Technology, Faculty of Pharmacy, University of Helsinki, Viikinkaari 5 E, 00014 Helsinki, Finland

${ }^{\ddagger}$ Biomedicum Imaging Unit, Faculty of Medicine, University of Helsinki, Haartmaninkatu 8, 00014 Helsinki, Finland

${ }^{\perp}$ Dodd-Walls Center for Photonic and Quantum Technologies, Department of Chemistry, University of Otago, Dunedin 9016, New Zealand

"Laboratory of Chemistry and Bioengineering, Tampere University of Technology, Korkeakoulunkatu 8, 33720 Tampere, Finland

\section{Supporting Information}

ABSTRACT: The tendency for crystallization during storage and administration is the most considerable hurdle for poorly watersoluble drugs formulated in the amorphous form. There is a need to better detect often subtle and complex surface crystallization phenomena and understand their influence on the critical quality attribute of dissolution. In this study, the interplay between surface crystallization of the amorphous form during storage and
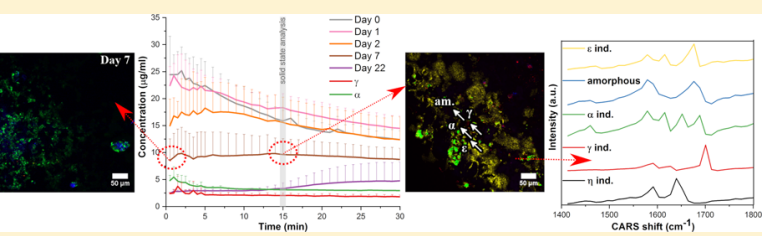
dissolution testing, and its influence on dissolution behavior, is analyzed for the first time with multimodal nonlinear optical imaging (coherent anti-Stokes Raman scattering (CARS) and sum frequency generation (SFG)). Complementary analyses are provided with scanning electron microscopy, X-ray diffraction and infrared and Raman spectroscopies. Amorphous indomethacin tablets were prepared and subjected to two different storage conditions $\left(30{ }^{\circ} \mathrm{C} / 23 \% \mathrm{RH}\right.$ and $\left.30{ }^{\circ} \mathrm{C} / 75 \% \mathrm{RH}\right)$ for various durations and then dissolution testing using a channel flow-through device. Trace levels of surface crystallinity previously imaged with nonlinear optics after 1 or 2 days of storage did not significantly decrease dissolution and supersaturation compared to the freshly prepared amorphous tablets while more extensive crystallization after longer storage times did. Multimodal nonlinear optical imaging of the tablet surfaces after $15 \mathrm{~min}$ of dissolution revealed complex crystallization behavior that was affected by both storage condition and time, with up to four crystalline polymorphs simultaneously observed. In addition to the well-known $\alpha$ - and $\gamma$-forms, the less reported metastable $\varepsilon$ - and $\eta$-forms were also observed, with the $\varepsilon$-form being widely observed in samples that had retained significant surface amorphousness during storage. This form was also prepared in the pure form and further characterized. Overall, this study demonstrates the potential value of nonlinear optical imaging, together with more established solid-state analysis methods, to understand complex surface crystallization behavior and its influence on drug dissolution during the development of amorphous drugs and dosage forms.

KEYWORDS: surface crystallization, amorphous, dissolution, nonlinear optics, indomethacin, polymorphism

\section{INTRODUCTION}

Conversion of the crystalline form of a drug into its amorphous form is one of the most promising enabling formulation approaches for the increasing number of poorly water-soluble drug molecules. The amorphous form, despite its thermodynamic physical instability and tendency for recrystallization, is often considered, as it offers a considerable solubility advantage over its crystalline counterpart.

In the early stages of amorphous drug development, solubility and dissolution are investigated. Experimentally, intrinsic dissolution tests ensure a constant surface area ${ }^{1}$ and thus potentially enable the evaluation of solid-state effects, while the particle size effects are minimized. In addition to the conventional rotating disk apparatus, different flow-through and channel flow systems have been developed. Theoretically, the solubility and dissolution advantage of amorphous drugs can be calculated on the basis of thermal properties. The calculated increases in solubility and dissolution are generally higher than those experimentally obtained. ${ }^{2}$ For instance, the experimentally determined solubility advantage of amorphous to crystalline $\gamma$-indomethacin is between $4.5^{2}$ and $4.9,^{3}$ whereas the predicted ratio at $25{ }^{\circ} \mathrm{C}$ is between 25 and $104 .^{2}$ It is believed that the driving force for dissolution can be predicted fairly well based on the thermodynamic calculations and that the experimentally determined values are often underestimated. ${ }^{2,3}$ One reason for underestimated experimental solubility and dissolution results is surface crystallization to less

Received: August 7, 2018

Revised: September 21, 2018

Accepted: September 24, 2018

Published: September 24, 2018 
soluble forms during dissolution (solution-mediated or direct solid-solid). Furthermore, during administration, crystallization of the amorphous form can also occur and lead to insufficient concentration of dissolved drug molecules available for absorption and a consequent lack of therapeutic effect. For these reasons, it is important to detect and understand crystallization processes during dissolution testing.

Coupling solid-state analysis with dissolution testing can provide understanding of the surface solid-state phenomena affecting the dissolution process. Glancing angle X-ray diffraction (XRD) has been used to monitor surface crystallization after dissolution of partially amorphous indomethacin compacts as a function of tablet depth. ${ }^{4}$ Imaging methods, such as polarized light microscopy (PLM) and ATRFTIR spectroscopic imaging, ${ }^{5}$ are also potentially attractive choices. However, the solid-state specificity of PLM is limited, and in the case of ATR-FTIR, the water complicates sampling and, depending on the selected region or peak of interest, can also interfere with analysis. ${ }^{5}$ Due to its insensitivity to water, Raman spectroscopy has been used in situ to monitor solidstate changes occurring during dissolution. ${ }^{6,7}$ However, conventional Raman systems, including with Raman probes, may not be sufficiently surface-specific to prevent the surface signal being overwhelmed by the signal from the core of the sample. ${ }^{6,7}$ In the case of a very thin crystalline layer forming on the surface of the amorphous sample, even Raman microscopy cannot always detect surface crystallinity. ${ }^{8}$ The relatively slow imaging speed of conventional Raman microscopy contributes to this challenge.

Nonlinear optical imaging, in particular involving coherent anti-Stokes Raman scattering (CARS) or sum frequency generation (SFG), is gaining interest in the solid-state analysis of pharmaceutics. ${ }^{9}$ As well as offering rapid contactless sampling with submicron lateral resolution, nonlinear analytical methods are highly surface sensitive due to their being inherently confocal, with the signal being generated only in the focal volume of the overlapping lasers when phase matching criteria are fulfilled. CARS is orders of magnitude faster than conventional Raman microscopy (based on spontaneous Raman scattering) and has been used to image crystal phase transformations associated with the dissolution of pharmaceutical tablets, ${ }^{10,11}$ in particular, solution-mediated hydrate formation. $^{12}$ Sum frequency generation (SFG), including second-harmonic generation (SHG), has proven powerful in detecting trace levels of crystallinity. ${ }^{13-17}$ SFG can differentiate amorphous from crystalline forms (as long as the crystals are not centrosymmetric), as only noncentrosymmetric crystals can generate a bulk SFG signal. However, SFG alone cannot separate different crystals that belong to the same classification in terms of centrosymmetry (although the magnitude of the SFG signal may be different). Multimodal nonlinear imaging, in which two or more nonlinear optical techniques are combined, can provide more specific and reliable analysis when the amorphous form and multiple polymorphs are present. In our recent study, ${ }^{18}$ the synergistic use of simultaneous CARS and SFG imaging allowed the distribution of the amorphous form and crystalline forms of the drug indomethacin to be imaged. The surface crystallization of the amorphous indomethacin tablets during storage was also imaged. ${ }^{18}$ Briefly, the tablets stored at lower humidity crystallized predominately to the $\gamma$-indomethacin, whereas the tablets stored at higher humidity crystallized mostly to the $\alpha$-form. Some small areas of the nondominant polymorph, not detected with ATR-FTIR or Raman spectroscopies, were also observed. Crystallization was also detected at an earlier stage with multimodal nonlinear optics than with ATR-FTIR and Raman spectroscopies.

Indomethacin is a widely studied drug with respect to its solid-state behavior and has been reported to exist in several polymorphic forms: $\alpha, \beta, \gamma, \delta, \varepsilon, \zeta, \eta, \tau$, as well as an unnamed form. ${ }^{19-24}$ Some of these forms have only been observed in quite specific conditions; for example, the $\zeta$ - and $\eta$-forms were detected after exposure of amorphous particles to $\mathrm{pH} 1.2$ buffer solution, while the $\tau$ form has been observed in semicrystalline dispersions. The $\beta$ form was later confirmed to be a benzene solvate. ${ }^{25}$ The crystal structures have been reported only for the $\alpha$ - and $\gamma$-polymorphs, although the powder XRD profiles for the other forms except $\varepsilon$ have been published. The $\varepsilon$-form is less well-characterized than the other polymorphs due to stability-related measurement difficulties. ${ }^{23}$ In addition to these crystalline forms, the amorphous form of indomethacin has been widely studied. Numerous studies have independently evaluated the dissolution ${ }^{26-28}$ and storageinduced $^{29-31}$ solid-state transformations of the amorphous form of indomethacin. However, studies exploring the relationship between the partial (surface) crystallization induced by storage and dissolution behavior are limited. ${ }^{32}$

In this study, multimodal nonlinear imaging (CARS and SFG), together with XRD and ATR-FTIR spectroscopy, were utilized to investigate the relationship between surface crystallization of amorphous indomethacin tablets, during both storage and dissolution, and dissolution behavior. Of particular interest was whether very early stage crystallization during storage, as detected earlier with nonlinear imaging, ${ }^{18}$ would affect surface crystallization during dissolution as well as dissolution behavior. The complex surface crystallization behavior of the freshly prepared and stored samples, including the simultaneous appearance of multiple solid-state forms and their distribution, was investigated.

\section{MATERIALS AND METHODS}

2.1. Sample Preparation. Amorphous indomethacin was prepared from the $\gamma$-form (Orion, Finland) by cooling the melt to room temperature. ${ }^{33}$ Once solidified, the sample was kept in a desiccator with phosphorus pentoxide for $30 \mathrm{~min}$, after which it was pulverized and compressed into tablets with a benchtop single punch press (Specac, UK). Flat-faced tablets were compressed with the same pressure of 1 ton and a dwell time of $30 \mathrm{~s}$. The tablets weighed $300 \pm 5 \mathrm{mg}$ and had a diameter of $13 \mathrm{~mm}$ and a height of $2 \mathrm{~mm}$. The same side of the tablet (upper side in the tablet press) was exposed to the dissolution medium and analyzed in all measurements. Freshly prepared amorphous indomethacin tablets were analyzed within a couple of hours and denoted as 0-day samples. To promote crystallization, the tablets were placed in open glass vials and stored at $30{ }^{\circ} \mathrm{C}$ at lower $(23 \%)$ or higher (75\%) relative humidity, obtained by using saturated salt solutions of potassium acetate and sodium chloride, respectively.

The $\gamma$-form (with a centrosymmetric P $\overline{1}$ crystal structure) was used as received. The $\alpha$-indomethacin form (with a noncentrosymmetric $\mathrm{P} 2{ }_{1}$ crystal structure) was obtained by antisolvent precipitation with Milli-Q water from a warm ethanol solution prepared from $\gamma$-indomethacin. ${ }^{25}$ The $\varepsilon$-form of indomethacin was prepared from a suspension of amorphous indomethacin in $\mathrm{pH} 6.8$ phosphate buffer. $^{23,34}$ The $\delta$-form was prepared from indomethacin methanolate. ${ }^{22}$ 
Tablets composed of pure $\alpha$-indomethacin were prepared in a similar manner as described for amorphous indomethacin. Attempts in making tablets composed from pure $\gamma$ indomethacin resulted in lamination and breakage in the dissolution cell sample cavity. Therefore, the initially amorphous tablets stored for 5 months at $30{ }^{\circ} \mathrm{C} / 23 \% \mathrm{RH}$ were used as pure $\gamma$-indomethacin tablets. Complete crystallization to the $\gamma$-indomethacin over a 5-month period was confirmed with $\mathrm{XRD}$, differential scanning calorimetry (DSC), and FTIR.

2.2. Intrinsic Dissolution Testing. Freshly prepared amorphous tablets, as well as those stored for $1,2,7$, or 22 days were subjected to dissolution testing in a custom-built channel flow system. The experimental setup was similar to that described by Peltonen et al. ${ }^{35}$ The tablets were inserted in the cavity in the middle of the cell such that only one surface was flushed with fresh dissolution medium. Tablets and the cavity had a diameter of $13 \mathrm{~mm}$ and thus a constant surface area of $1.33 \mathrm{~cm}^{2}$. The peristaltic pump (Watson Marlow $505 \mathrm{U})$, channel flow cell, and reservoir with dissolution medium were connected with silicone tubing in an open loop manner. This ensured the constant availability of fresh buffer and maintenance of true sink conditions. The flow was kept constant at $9 \mathrm{~mL} / \mathrm{min}^{36}$ This rate has earlier been found to simulate the (axial) velocity of the intestine best. ${ }^{37}$ Solution samples were collected downstream every $30 \mathrm{~s}$ for the first 5 min and then in 1 min intervals until $30 \mathrm{~min}$. The absorbance of unfiltered samples ${ }^{33}$ was measured at $318 \mathrm{~nm}^{1}$ with a 1600PC UV-Vis spectrophotometer (VWR, China). Every dissolution experiment was performed at least in triplicate. Phosphate buffer with a $\mathrm{pH}$ of 6.8 was used as the aqueous medium. A series of standard solutions of indomethacin in $\mathrm{pH}$ 6.8 phosphate buffer with concentrations ranging from 0.5 to $40 \mu \mathrm{g} / \mathrm{mL}$ was used to construct a calibration curve for quantification. As the tablets remained whole at the end of the experiment, the tablet surface exposed to the liquid medium could subsequently be further analyzed. All solid-state measurements on tablets subjected to dissolution testing were performed after $15 \mathrm{~min}$ of dissolution testing. Prior to solid-state analysis, the tablets' surfaces were blotted with lens cleaning tissue paper and removed from the channel flow cell.

2.3. Scanning Electron Microscopy (SEM). Surface sections of the tablets were mounted on double-sided carbon tape and sputter coated with platinum. The micrographs were obtained with a FEI Quanta 250 field emission gun SEM (FEI, Hillsboro, USA) microscope using a high vacuum and a voltage of $10 \mathrm{kV}$.

2.4. X-ray Diffractometry (XRD). Diffractograms were collected in reflection mode using a Malvern Panalytical Empyrian (PANalytical B.V. Almelo, The Netherlands) instrument with $\mathrm{Cu} \mathrm{K} \alpha$ radiation $(\lambda=1.5406)$ and $\mathrm{a}$ divergence slit of $0.76 \mathrm{~mm}$. The generator voltage was $45 \mathrm{kV}$, and the tube current $40 \mathrm{~mA}$. Data were collected in a $2 \theta$ scan range of 5 to $50^{\circ}$ with a step size of $0.013^{\circ}$. The tablets were rotated during the measurements.

2.5. Fourier Transform Infrared Spectroscopy (FTIR). FTIR analysis was performed using a single-reflection MIRacle attenuated total reflectance (ATR) crystal (Pike Technologies, Wisconsin, USA) with a Vertex 70 spectrometer (Bruker Optics, Ettlingen, Germany). Measurements were collected using OPUS 5.5 (Bruker Optics, Ettlingen, Germany) software. Each spectrum consisted of an average of 256 scans with a spectral resolution of $4 \mathrm{~cm}^{-1}$. The obtained spectra were standard normal variance (SNV) corrected prior to principal component analysis (PCA) in the spectral range of 1400-1800 $\mathrm{cm}^{-1}$ using Simca software V10.5 (Umetrics, Umea, Sweden).

2.6. Multimodal Nonlinear Optical Imaging. Multimodal nonlinear imaging was performed with a Leica SP8 CARS microscope (Leica, Wetzlar, Germany). The CARS excitation source was a solid-state $\mathrm{Nd}: \mathrm{YVO}_{4}$ laser. The Stokes (fundamental) laser line at $1064.5 \mathrm{~nm}$ was temporally and spatially overlapped with the pump/probe beam. The wavelength of the pump line was tuned by an optical parametric oscillator (OPO). Combined incident beams were directed into an inverted microscope with an IR corrected $25 \times, 0.95$ NA water immersion objective. The signal was collected in epi (backscattered) mode. For narrowband (single line) imaging and CARS spectral scans, photomultiplier tube (PMT) detectors were used, while for the second-order nonlinear spectra (including SFG), hybrid detectors (HyD) were used. Bright field images were collected in reflection mode using a $633 \mathrm{~nm} \mathrm{HeNe}$ laser and PMT detector in the 623-643 nm range. The step size for the CARS spectral measurements was $1 \mathrm{~nm}$ (corresponding to a spectral resolution of approximately $12 \mathrm{~cm}^{-1}$ ), whereas for the second-order spectral scans, the step size was $10 \mathrm{~nm}$.

Three imaging modalities were used for the same field of view: (i) CARS spectral scans in the range of 1413-1800 $\mathrm{cm}^{-1}$, (ii) second-order nonlinear spectral scans in the range of 400 to $700 \mathrm{~nm}$, and (iii) a combination of narrowband CARS and SFG imaging. The combination images represent overlays of three channels: a single CARS line at $1701 \mathrm{~cm}^{-1}$ in red $(\gamma$ indomethacin), a single CARS line at $1676 \mathrm{~cm}^{-1}$ in blue (amorphous indomethacin), and a third channel representing the SFG signal (all noncentrosymmetric crystals). Potential two-photon excited fluorescence background signal interference in the narrowband images was minimized by subtracting the images obtained by one-laser excitation (pump laser only). Images represent the maximum projections of single-plane optical images at $2 \mu \mathrm{m}$ steps in the $z$-direction. This step size was selected, as the instrument axial resolution is $2.5 \mu \mathrm{m}$. The total depth of the maximum projection images obtained in this manner was in the range of 20 to $52 \mu \mathrm{m}$. The lateral image size was $512 \times 512$ pixels covering a $465 \times 465 \mu \mathrm{m}$ area with images being collected with a line average of 4 and pixel dwell time of $1.2 \mu \mathrm{s}$. At least three different spots of each tablet were analyzed. To resolve SFG signal originating from different SFG-active polymorphs, the characteristic CARS peaks from the same regions were analyzed using Fiji ImageJ 1.51. False coloring (green and yellow) was assigned based on the relative intensity ratios of the CARS peaks at 1652 and $1676 \mathrm{~cm}^{-1}$. The regions having SFG activity and a CARS peak at $1652 \mathrm{~cm}^{-1}$ characteristic for $\alpha$-indomethacin were colored green, while those having SFG activity and a CARS peak at $1676 \mathrm{~cm}^{-1}$ characteristic for $\varepsilon$-indomethacin were colored yellow.

\section{RESULTS}

3.1. Dissolution Behavior. 3.1.1. Pure Amorphous and Reference Crystalline Forms. As shown in Figure 1, the maximum dissolved concentration for the 0-day sample was reached after 2 min of dissolution testing and was around 12fold higher than that of the reference $\gamma$-crystalline form of indomethacin, which stabilized at around $2 \mu \mathrm{g} / \mathrm{mL}$. Supersaturation was followed by a concentration drop, indicating solution-mediated crystallization. The crystalline $\alpha$-form 


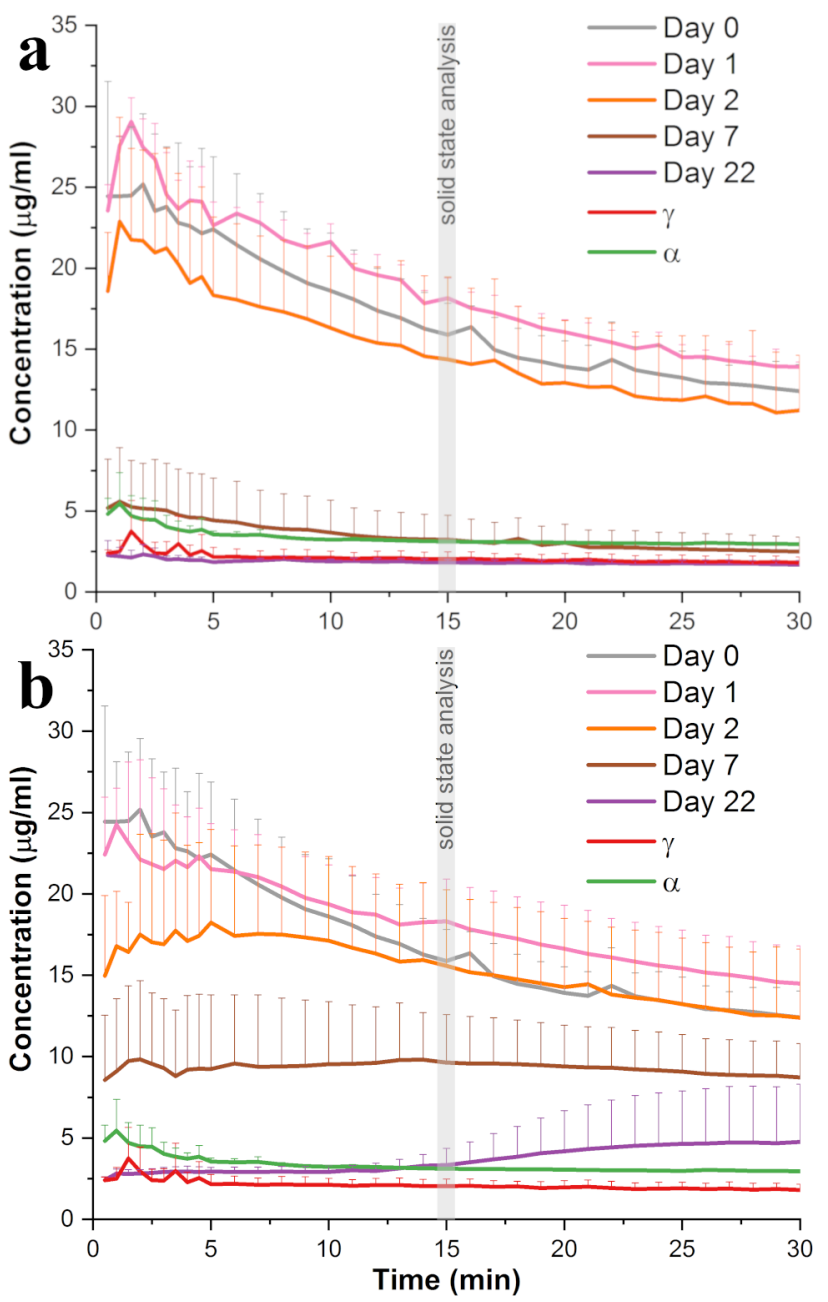

Figure 1. Dissolution profiles (drug release over time) for samples stored at (a) $30{ }^{\circ} \mathrm{C} / 23 \% \mathrm{RH}$ and (b) $30{ }^{\circ} \mathrm{C} / 75 \% \mathrm{RH}$. Profiles of the pure amorphous tablet (day 0 ) and reference crystalline $\gamma$ - and $\alpha$ forms are shown for comparison. Profiles are generated with mean values of a minimum of three measurements. Error bars represent plus one standard deviation. exhibited a slightly increased concentration compared to the $\gamma$ form (around $3 \mu \mathrm{g} / \mathrm{mL}$ ).

Crystallization on the surface of a dissolving tablet can increase the effective surface area available for dissolution. ${ }^{38}$ Such an effect could be of greater influence for samples with higher levels of crystallization where the increase in surface area is greater. This can potentially explain why the dissolution profiles were not approaching those of the reference crystalline forms toward the end of the measurements. Another potential reason is that metastable polymorphs, more soluble than the $\alpha$ or $\gamma$-forms, were crystallizing on the tablet surface.

3.1.2. Stored Samples. Amorphous indomethacin samples were kept in controlled storage conditions for 22 days, and their dissolution properties were periodically monitored. The largest differences between the samples in Figure 1 can be seen within the first $15 \mathrm{~min}$. The maximum drug concentrations decreased upon increased storage time. At the beginning of the experiments, the dissolution profiles of the 1-day and 2-day samples were still largely similar to that of the freshly prepared tablets and faster than those of the samples stored for longer periods, although the 2-day sample stored at $30{ }^{\circ} \mathrm{C} / 75 \% \mathrm{RH}$ exhibited a slightly lower maximum concentration. The most pronounced effects of storage time and conditions can first be seen at 7 days of storage, when the maximum and subsequent concentrations were substantially lower. This was especially the case for tablets stored at $30{ }^{\circ} \mathrm{C} / 23 \% \mathrm{RH}$, whose achieved concentrations were around two times lower than those of the tablets stored at $30{ }^{\circ} \mathrm{C} / 75 \% \mathrm{RH}$. The dissolution profiles of the 22 -day tablets stored at $30{ }^{\circ} \mathrm{C} / 23 \% \mathrm{RH}$ closely resembled those of the reference $\gamma$-form. The profiles of the 22 -day tablets stored at $30{ }^{\circ} \mathrm{C} / 75 \% \mathrm{RH}$ were between or similar to those of the reference $\alpha$ - and $\gamma$-crystalline forms.

3.2. Visual Appearance. A change in surface color upon dissolution, indicating crystallization, was observed for many of the samples. This was visible especially when tablets were broken: a thin white layer that had formed on the surface exposed to the dissolution medium was clearly observed. The rest of the tablet remained yellow, which is characteristic of the amorphous form. A dissolution-induced change was less pronounced or not present for the samples stored for longer periods of time (7 and 22 days) at lower humidity, as they

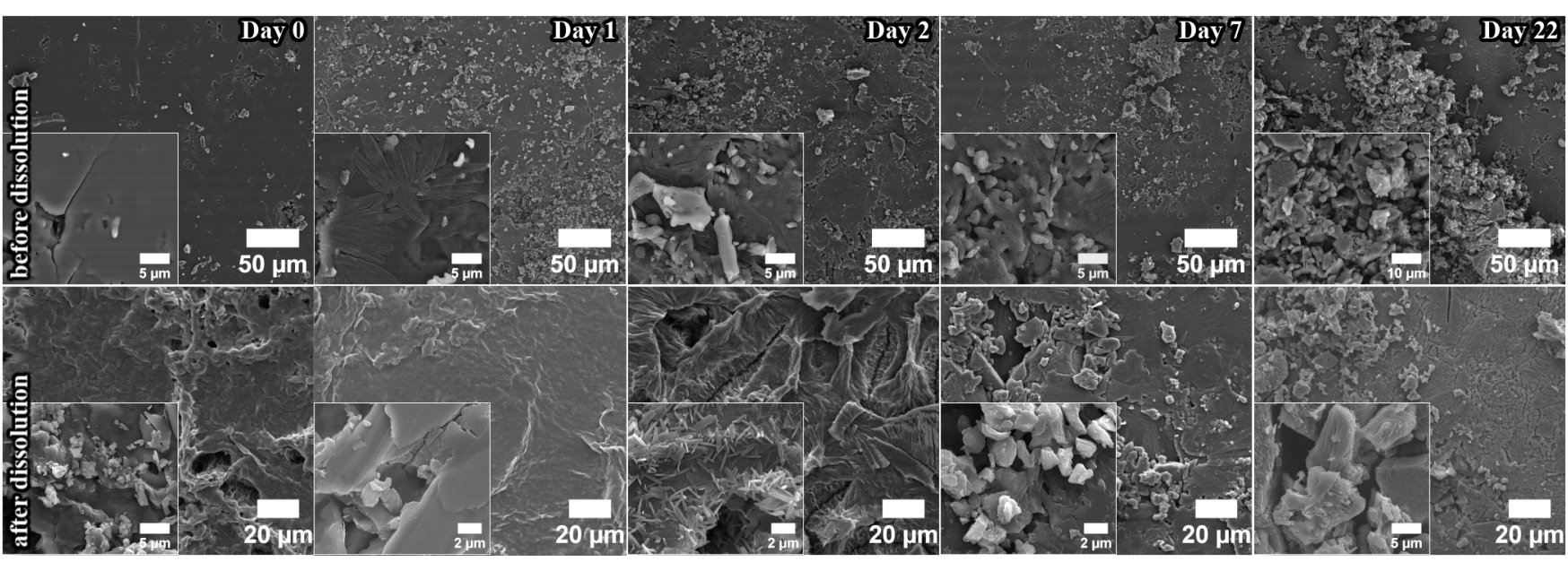

Figure 2. SEM micrographs of an amorphous indomethacin tablet (day 0, first column) and tablets stored for $1,2,7$, and 22 days (from left to right) at $30{ }^{\circ} \mathrm{C} / 23 \% \mathrm{RH}$ before dissolution testing (top row). SEM images of the tablets prepared and stored in the same manner after $15 \mathrm{~min}$ of intrinsic dissolution testing (bottom row). 


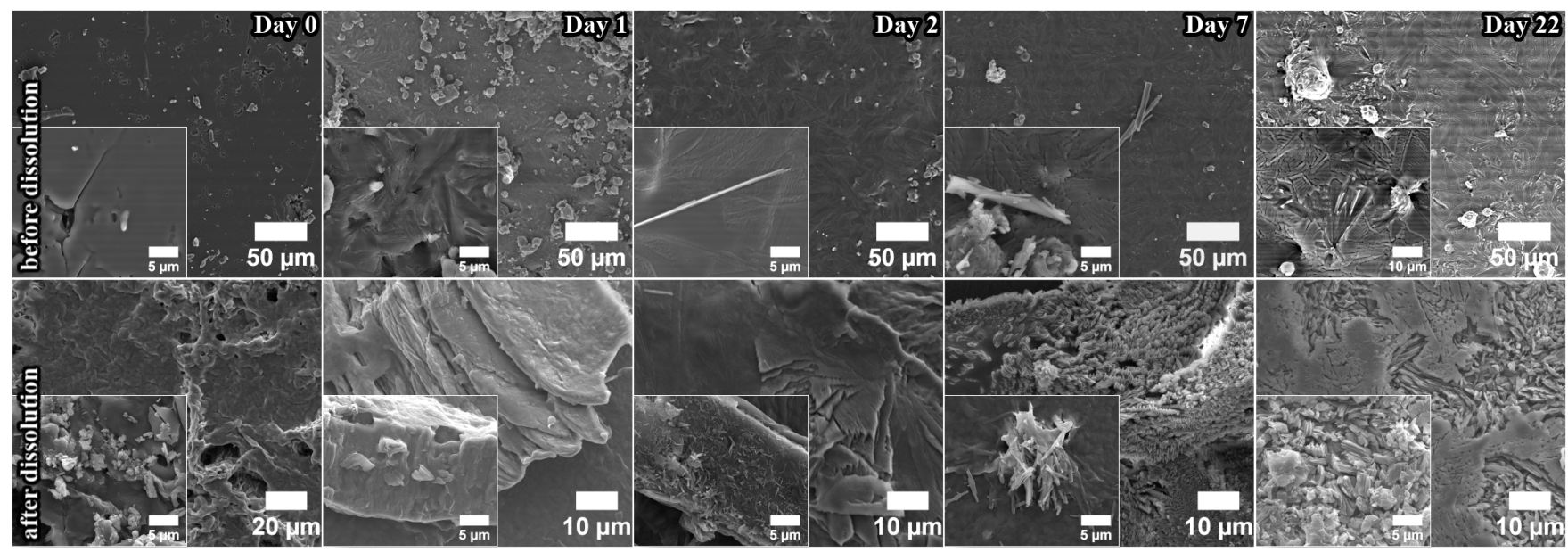

Figure 3. SEM micrographs of an amorphous indomethacin tablet (day 0, first column) and tablets stored for 1, 2, 7, and 22 days (from left to right) at $30^{\circ} \mathrm{C} / 75 \% \mathrm{RH}$ before dissolution testing (top row). SEM images of the tablets prepared and stored in the same manner after $15 \mathrm{~min}$ of intrinsic dissolution testing (bottom row).

were already relatively white and presumably relatively crystalline throughout the tablet prior to dissolution testing.

3.3. Surface Morphology. The morphology of fresh and stored tablet surfaces after $15 \mathrm{~min}$ of dissolution testing was compared to the surfaces before dissolution testing using SEM (Figures 2 and 3). The surface of the freshly prepared tablet previously confirmed as amorphous was quite smooth. ${ }^{18}$ Surfaces of tablets stored at $30{ }^{\circ} \mathrm{C} / 23 \% \mathrm{RH}$ prior to dissolution featured plate-like particles, and their surface coverage increased over time. The storage-induced changes in surface morphology were slower for the tablets stored at 30 ${ }^{\circ} \mathrm{C} / 75 \% \mathrm{RH}$, and many observed particles on these tablets had needle-like morphology. ${ }^{18}$

After $15 \mathrm{~min}$ of dissolution, the surface of the 0-day tablet had changed; crystal-like particles were observed in addition to smooth-looking areas. The 1-day samples after dissolution were similar to the freshly prepared samples after dissolution and also had some largely smooth regions. For both storage conditions, the 2-day samples had more needle-shaped particles after dissolution. The needles were usually $1-2 \mu \mathrm{m}$ in size, with the largest observed needles being $40 \mu \mathrm{m}$ long. The morphologies of the samples stored for the same times at different storage conditions after dissolution testing became strikingly different at day 7 . The surfaces of tablets stored at 30 ${ }^{\circ} \mathrm{C} / 23 \% \mathrm{RH}$ was covered with plate-like particles, mostly $1-2$ $\mu \mathrm{m}$ in size, with some up to about $5 \mu \mathrm{m}$. A few needle-shaped structures were also observed, but their number was considerably lower. In contrast, the surfaces of tablets stored at $30{ }^{\circ} \mathrm{C} / 75 \% \mathrm{RH}$ had many needle-like particles with some arranged in a spiral-like morphology (Figure 3, day 7 after dissolution). Most of the needles were between 5 and $10 \mu \mathrm{m}$ long, although the largest was $100 \mu \mathrm{m}$ in length. This difference for the two different storage conditions remained pronounced for the 22-day samples after dissolution. The largest observed plate-like particles for samples stored at lower humidity were $20 \mu \mathrm{m}$. Needle shaped particles for samples stored at higher humidity appeared more fused together over larger areas, and the majority of the individual particles were about $5 \mu \mathrm{m}$ in length.

Crystals of $\gamma$-indomethacin generally have a plate-like morphology, whereas $\alpha$-indomethacin crystals are needle-like with growth in the form of fibrous/spherulitic structures. ${ }^{39}$
While the SEM images are suggestive of storage time- and condition-dependent crystallization for at least these two forms during dissolution, more specific solid-state analysis was performed with multimodal nonlinear optical imaging, together with XRD and ATR-FTIR spectroscopy.

3.4. Solid-State Analysis. Solid-state analysis was performed off-line by XRD, ATR-FTIR, and CARS/SFG upon 15 min of dissolution.

3.4.1. Freshly Prepared Amorphous Indomethacin (Day $0)$. Prior to dissolution, the freshly prepared indomethacin tablet was amorphous according to XRD (Figure 4). Even
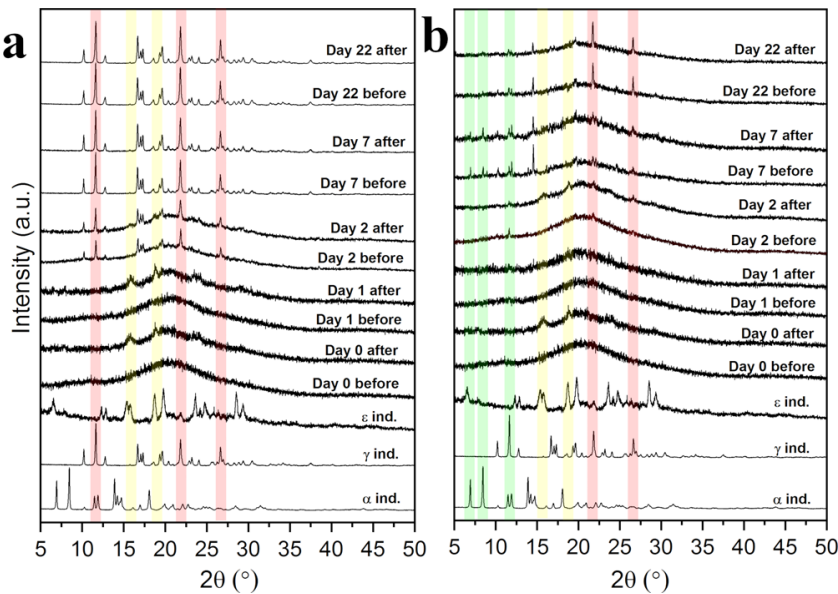

Figure 4. XRD diffractograms of tablets before and after $15 \mathrm{~min}$ of dissolution testing: (a) storage at $30{ }^{\circ} \mathrm{C} / 23 \% \mathrm{RH}$ and (b) storage at $30{ }^{\circ} \mathrm{C} / 75 \%$ RH. Diffractograms of the $\alpha$-, $\gamma$-, and $\varepsilon$-forms of indomethacin are shown for comparison, and some of their characteristic peaks are marked in green, red, and yellow, respectively. The $\varepsilon$-form was measured in transmission mode using a powder, while the $\alpha$ - and $\gamma$-forms were measured in reflection with tablets.

though any potential remaining surface moisture after tablet blotting could partially attenuate the XRD signal, after $15 \mathrm{~min}$ of dissolution testing, we were able to see the appearance of two small diffraction peaks at 15.8 and $18.8^{\circ}$ above the amorphous halo. This indicated partial crystallization. These two peaks did not correspond to those of previously published $\mathrm{X}$-ray diffractograms of indomethacin polymorphs $\left(\alpha, \gamma,{ }^{19,25}\right.$ 
$\delta,^{22} \zeta, \eta^{23}$ and $\left.\tau^{24}\right)$. The $\varepsilon$-form of indomethacin was also prepared in the present study, and its XRD pattern is presented and attributed to this form for the first time (Figure 4). The two peaks from the tablet after dissolution testing correspond to this diffraction pattern of the $\varepsilon$-form (marked in yellow in Figure 4). The diffractogram of the $\varepsilon$-form also had considerable similarity with the unnamed form of indomethacin reported by Lin. $^{21}$

ATR-FTIR spectroscopy, which is more surface-specific than XRPD (sampling depth limited to approximately 1 to $2 \mu \mathrm{m}$ ) was also performed (Figure S1). Based on the PCA of the spectra (Figure 5), the same samples (after $15 \mathrm{~min}$ of
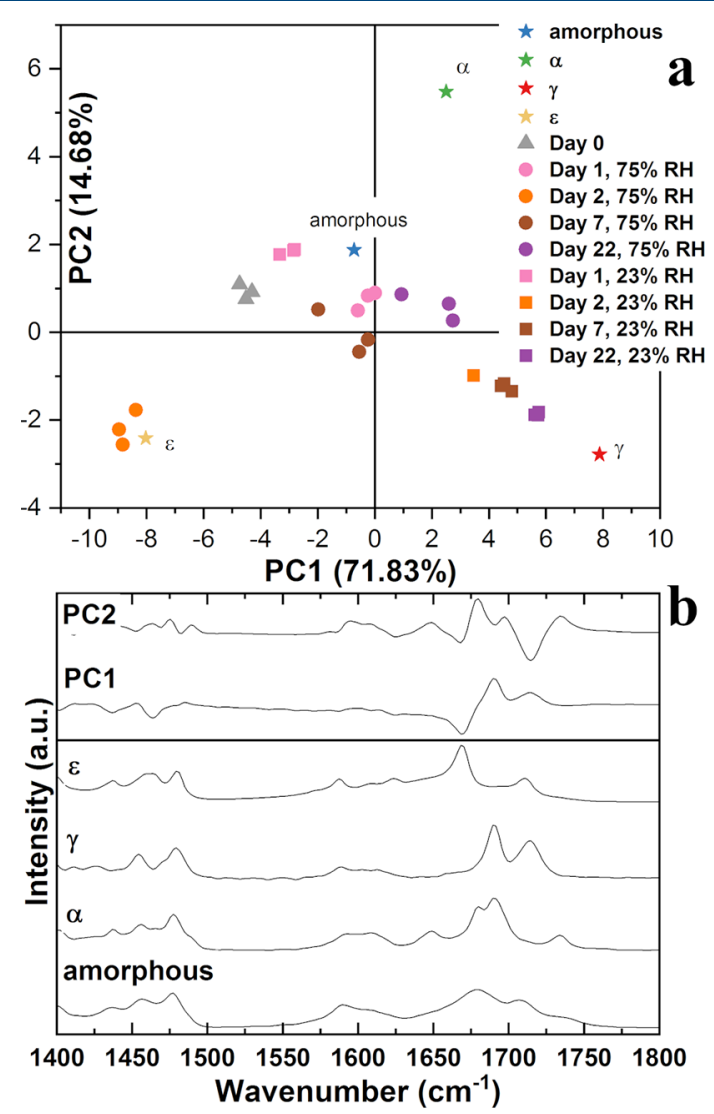

Figure 5. PCA scores plot of the ATR-FTIR spectra of the amorphous (day 0 ) and all stored samples after 15 min of dissolution testing (a); loadings and reference IR spectra of different solid-state forms of indomethacin (b).

dissolution testing) were most similar to the amorphous form even though their score values slightly approached that of the crystalline $\varepsilon$-form. This indicates some limited surface coverage with the $\varepsilon$-form.

To obtain more precise information about the dissolutioninduced surface distribution of the $\varepsilon$-form and any other potential minority species not detected with XRD and ATRFTIR, multimodal nonlinear imaging was performed. No SFG or CARS signals indicative of crystallization were detected on the surface of the freshly prepared amorphous tablets prior to dissolution testing (Figures $6 \mathrm{a}$ and $7 \mathrm{a}$ ), further confirming their amorphous nature. SFG has previously been shown to be very sensitive in detecting compression-induced crystallization to polymorphs with a noncentrosymmetric structure. ${ }^{16}$ In contrast, signs of extensive crystallization after dissolution testing could be seen in a strong SFG signal (in yellow in
Figures $6 \mathrm{~b}$ and $7 \mathrm{~b})$. As these signals are only generated in the absence of centrosymmetry (amorphous forms do not fulfill this criterion but noncentrosymmetric crystals do), their presence was a definite sign of the initially amorphous tablet surface crystallizing during dissolution. The CARS spectral scans of the same area of view provided further insights into the polymorphism. The spectra from the SFG-active regions featured peaks at 1579, 1615, and $1676 \mathrm{~cm}^{-1}$ (Figures $6 \mathrm{~d}$ and $7 \mathrm{~d}$ ), which correspond to the reference CARS spectrum of the $\varepsilon$-form of indomethacin. No other minority species were detected.

3.4.2. Tablets after Storage at Lower Humidity $\left(30^{\circ} \mathrm{C} /\right.$ $23 \% R H)$. XRD analysis of the 1-day tablet stored at lower humidity prior to dissolution testing showed only an amorphous halo (Figure 4). After $15 \mathrm{~min}$ of dissolution, however, two small diffraction peaks (at 15.8 and $18.8^{\circ}$ marked in yellow) above the amorphous halo could be observed. These peaks corresponded to the $\varepsilon$-form of indomethacin. For the 2-, 7 -, and 22-day samples, almost no differences between the diffraction patterns for the corresponding before and after dissolution samples were observed. The 2-day samples exhibited an amorphous halo with some characteristic diffraction peaks of $\gamma$-indomethacin. The amorphous halo was not observable for the 7- and 22-day samples, with the diffractograms suggesting they were composed exclusively of $\gamma$ indomethacin already prior to dissolution.

The ATR-FTIR spectra of the tablet surfaces after storage at lower humidity (before dissolution) confirmed crystallization to the $\gamma$-form of indomethacin previously reported after 5 days of storage. ${ }^{18}$ The ATR-FTIR spectra of the tablets upon dissolution were consistent with crystallization to the $\varepsilon$ - and $\gamma$ forms observed with XRD (Figure S1). In the PCA scores plot (Figure 5), the post-dissolution tablets stored at lower humidity (marked with squares) gradually moved away from the amorphous and $\varepsilon$-forms and approached the $\gamma$-form as storage time increased. Thus, a post-dissolution trend from the $\varepsilon$ - to $\gamma$-form as a function of storage time was observed.

Nonlinear optical imaging allowed a more detailed analysis of the surface crystallization behavior and distribution of the solid-state forms. Unlike for the freshly prepared samples, CARS spectra characteristic of $\gamma$-indomethacin were extracted from the images of all samples stored at lower humidity and the corresponding samples that underwent dissolution testing (Figure 6e). Thus, the spectra confirmed $\gamma$-indomethacin crystals after $15 \mathrm{~min}$ of dissolution as early as after 1 day of storage (Figure $6 \mathrm{~b}$ ), and a few $\gamma$ crystals were also observed predissolution. ${ }^{18}$ This crystallization to the $\gamma$-form at day 1 , both with the pre and postdissolution samples, was not detectable with the XRD and ATR-FTIR setups used. The increase in the surface area covered with $\gamma$-indomethacin crystals (in red) can be seen in images of tablets stored for increasing amounts of time both before and after dissolution.

The presence of the SFG-active $\varepsilon$-form with characteristic CARS peaks at 1579,1615 , and $1676 \mathrm{~cm}^{-1}$ (Figure $6 \mathrm{~d}$ ) was also observed for 1-day samples' post-dissolution (but not before dissolution). Unlike XRD and ATR-FTIR, with which the $\varepsilon$-indomethacin was detected only at day 1 of postdissolution, areas covered with the $\varepsilon$-form were observed with nonlinear imaging for all the samples stored at lower humidity (including 0-day) except for the 22-day sample. The areas covered with the $\varepsilon$-form post-dissolution decreased over storage time, while the $\gamma$-indomethacin coverage increased. Relatively large amorphous regions could still be seen on day 2, 



Figure 6. CARS and SFG overlay images of samples stored at $30{ }^{\circ} \mathrm{C} / 23 \% \mathrm{RH}$ before (a) and after (b) 15 min of dissolution testing at $\mathrm{pH} 6.8$. CARS spectra in the range of $1413-1800 \mathrm{~cm}^{-1}$ from selected regions marked by arrows plotted with reference spectra of amorphous, $\varepsilon$-, and $\gamma$ indomethacin $(\mathrm{c}-\mathrm{e})$. Overlay images $(\mathrm{a}, \mathrm{b})$ represent overlays of three channels: single CARS line at $1701 \mathrm{~cm}^{-1}$ in red $(\gamma$-indomethacin), single CARS line at $1676 \mathrm{~cm}^{-1}$ in blue (amorphous indomethacin), and a third channel representing the SFG signal (all noncentrosymmetric crystals) in green and yellow. The separation between green and yellow regions is based on the intensity ratios of CARS peaks at 1652 and $1676 \mathrm{~cm}^{-1}$ so that the regions having SFG activity and a CARS peak at $1652 \mathrm{~cm}^{-1}$ are colored green ( $\alpha$-indomethacin), and the regions having SFG activity and a CARS peak at $1676 \mathrm{~cm}^{-1}$ are colored yellow ( $\varepsilon$-indomethacin). Panel (a) has been reprinted with permission from Novakovic et al. ${ }^{18}$ Copyright 2017 American Chemical Society. am = amorphous.

whereas from 7 days onward, they were notably decreased/ absent.

Solid-state-specific visualization of tablet surfaces with nonlinear imaging in addition revealed some specific distribution patterns. This was most apparent with cracks present on the tablet surfaces at days 1 and 2-crystallization to the $\varepsilon$-form was concentrated in the vicinity of these cracks.

3.4.3. Tablets after Storage at Higher Humidity $\left(30^{\circ} \mathrm{C} /\right.$ $75 \% R H)$. All samples stored at higher humidity, both before and after dissolution, were at least partially amorphous according to XRD (Figure 4). An amorphous halo characterized the 1-day sample both prior and subsequent to dissolution. The 2-, 7-, and 22-day samples had some $\gamma$ indomethacin diffraction peaks, while the 7- and 22-day samples also exhibited diffraction peaks characteristic of $\alpha$ indomethacin.

Crystallization to the $\alpha$-form of indomethacin after 22 days of storage at higher humidity (before dissolution) was confirmed with ATR-FTIR spectroscopy previously. ${ }^{18}$ As can be seen from the PCA scores plot of the ATR-FTIR spectra, the 1-, 7-, and 22-day tablets stored at higher humidity that underwent dissolution testing (marked with circles in Figure 5) were mostly located between the reference solid-state forms of indomethacin, indicating that multiple forms were present simultaneously. The 2-day sample, however, was similar to $\varepsilon$ indomethacin.

Multiple polymorphs were simultaneously detected with multimodal CARS and SFG imaging. After storage and prior to dissolution, crystallization to $\alpha$-indomethacin was predominantly observed at higher humidity. This was accompanied by some traces of the $\gamma$-form (Figure 7a). ${ }^{18}$ Upon dissolution, in contrast to XRD and ATR-FTIR, crystallization was evident for the 1-day sample as seen by the strong SFG signals in yellow (Figure $7 \mathrm{~b}$ ). At days 1 and 2 after dissolution, the majority of the observed crystals (in yellow) were $2-3 \mu \mathrm{m}$ in size. With the samples stored for 7 and 22 days after dissolution, the crystalline areas appeared slightly larger, with individual crystals (in green) equal to or exceeding $10 \mu \mathrm{m}$. The CARS spectra indicate that there were five solid-state forms after dissolution testing. Similar to the samples stored at lower 



Figure 7. CARS and SFG overlay images of samples stored at $30^{\circ} \mathrm{C} / 75 \% \mathrm{RH}$ before (a) and after 15 min of dissolution testing at $\mathrm{pH} 6.8$ (b). CARS spectra in the range of $1413-1800 \mathrm{~cm}^{-1}$ from selected regions marked by arrows $(\mathrm{c}-\mathrm{f})$ plotted with reference spectra of amorphous, $\varepsilon$-, $\alpha$-, and $\gamma$-indomethacin. Overlay images $(\mathrm{a}, \mathrm{b})$ represent overlays of three channels: single CARS line at $1701 \mathrm{~cm}^{-1}$ in red $(\gamma$-indomethacin), single CARS line at $1676 \mathrm{~cm}^{-1}$ in blue (amorphous indomethacin), and a third channel representing the SFG signal (all noncentrosymmetric crystals) in green and yellow. The separation between green and yellow regions is based on the intensity ratios of CARS peaks at 1652 and $1676 \mathrm{~cm}^{-1}$ so that the regions having SFG activity and the CARS peak at $1652 \mathrm{~cm}^{-1}$ are colored green ( $\alpha$-indomethacin), and the regions having SFG activity and CARS peak at $1676 \mathrm{~cm}^{-1}$ are colored yellow ( $\varepsilon$-indomethacin). Panel (a) has been reprinted with permission from Novakovic et al. ${ }^{18}$ Copyright 2017 American Chemical Society. am = amorphous.

humidity, the areas with characteristic CARS peaks at 1579, 1615 , and $1676 \mathrm{~cm}^{-1}$ and SFG activity assigned to the $\varepsilon$ indomethacin were observed for all the postdissolution samples stored at higher humidity except for day 22 (Figure $7 \mathrm{~d})$. CARS spectra of $\alpha$-indomethacin were observed from 2 days of storage onward (Figure 7e). Traces of the $\alpha$-form were also detected for the 1-day sample (Figure S2). The presence of $\gamma$-indomethacin was confirmed in all stored samples after dissolution testing (Figure $7 \mathrm{f}$ ). Interestingly, the regions covered with the $\gamma$-form were largest after 22 days of storage. This finding is consistent with the XRD data, where the strong peaks of the $\gamma$-form were observed in addition to the less intense peaks corresponding to $\alpha$-indomethacin (Figure 4b).

Areas having SFG activity and two strong sharp CARS peaks at 1591 and $1640 \mathrm{~cm}^{-1}$ were clearly observed after dissolution only on day 7 (Figure S3). These areas were found only in traces. Based on the Raman spectra of indomethacin polymorphs available in the literature, ${ }^{23,24}$ it is likely that this polymorph is the $\eta$-form (with benzoyl $\mathrm{C}=\mathrm{O}$ stretching at $1642 \mathrm{~cm}^{-1}$ in the Raman spectra ${ }^{23}$ ).

3.4.4. Reference Raman and CARS Spectra. For reference, CARS and Raman spectra of many of the different solid-state forms of indomethacin are provided. Figure 8 shows the CARS spectra of the $\gamma_{-}, \alpha$-, $\delta$-, amorphous, $\varepsilon_{\text {-, }}$ and $\eta$-indomethacin forms as well as the Raman spectra of these forms plus the $\zeta$ form. As the CARS and Raman spectral features arise from the same molecular vibrations, they are uniquely related to one another. ${ }^{18,40}$ Carbonyl stretching of the carboxylic acid and benzoyl $\mathrm{C}=\mathrm{O}$ groups of indomethacin feature solid-statespecific bands between 1500 and $1800 \mathrm{~cm}^{-1}$. The CARS and Raman spectra for each solid-state form match one another. Detailed FTIR and Raman band assignments have been provided elsewhere. ${ }^{23,41}$ The lower spectral resolution of the CARS spectra compared to the Raman spectra explains why some smaller peaks or shoulders visible in the Raman spectra disappear or are merged into larger peaks in the CARS spectra.

\section{DISCUSSION}

The concentration versus time dissolution profiles for the freshly prepared amorphous samples show behavior typical of amorphous indomethacin with different stages able to be identified. ${ }^{8,27}$ As the dissolution rate is directly proportional to the solubility of the drug (for a constant surface area), the solubility of the solid-state form at the surface of the compact dictates the release rate. Thus, after supersaturation is achieved and the solution-mediated crystallization covers the exposed 


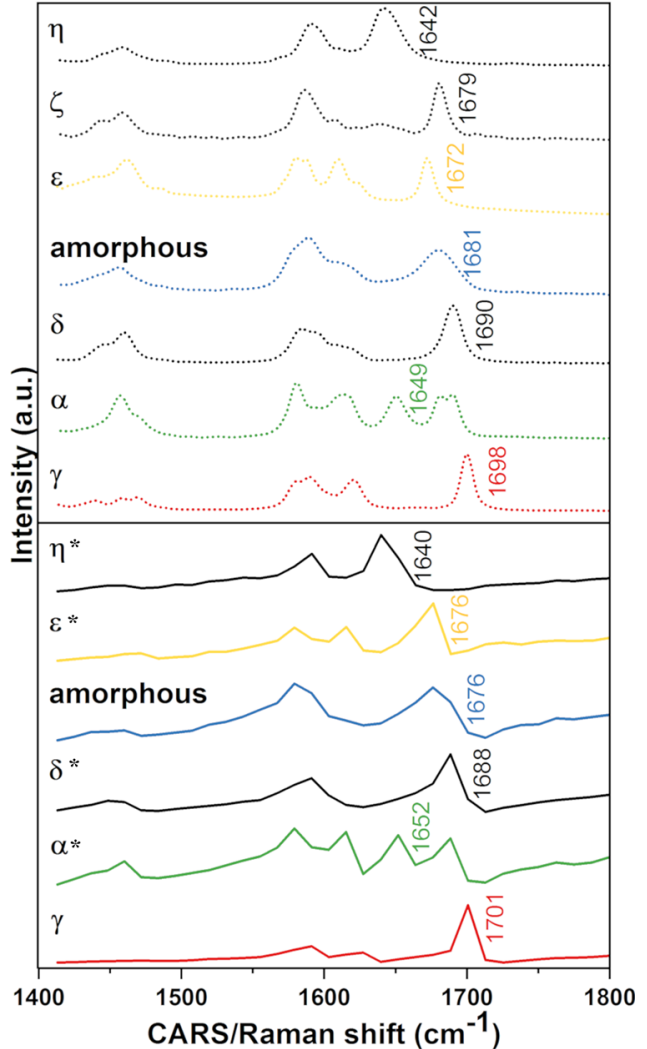

Figure 8. CARS (solid lines) and Raman (dotted lines) spectra of indomethacin solid-state forms. Raman spectra (except for the $\varepsilon$ form) are reproduced with permission from ref 23. The CARS spectral resolution is $12 \mathrm{~cm}^{-1}$, while the Raman spectral resolution is $4 \mathrm{~cm}^{-1}$. All CARS spectra are measured from the prepared reference forms, except for the spectrum of the $\eta$-form, which was recorded at the tablet surface. Polymorphs marked with asterisk are SFG-active.

tablet surface, the intrinsic dissolution rate decreased. ${ }^{7}$ The relatively large variability between the replicates for partially amorphous samples as observed in this study has been observed before. ${ }^{4}$ This has been attributed to the differences in tablet microstructure and preferred orientation effects. Batchto-batch variation of dissolution behavior of an amorphous formulation of tacrolimus, due to different crystallization kinetics, has also been reported. ${ }^{42}$
The decrease in supersaturated concentrations of the stored samples compared to the pure amorphous indomethacin indicates that retardation of dissolution is induced by crystallization during storage. In our previous study, ${ }^{18}$ early stage crystallization during storage was detected with nonlinear imaging already at days 1 and 2, which was at an earlier stage than with ATR-FTIR and Raman microscopy. Here, we have shown that this very early stage crystallization does not significantly affect the dissolution performance with these samples, as the final concentrations were similar for 0-, 1-, and 2-day samples at both storage conditions. Early stage crystallization could, however, be important and reduce drug dissolution in other samples or dissolution conditions, if these crystals were to act as seeds and promote solution-mediated growth of less soluble solid-state forms.

The crystallization of freshly prepared amorphous indomethacin upon dissolution has been quite extensively studied. Conversion to the $\alpha$-polymorph during dissolution has previously been observed with Raman ${ }^{26}$ and in situ Raman spectroscopy. ${ }^{6,27}$ The $\alpha$-form has been found to dominate in precipitates from supersaturated amorphous indomethacin solutions formed in ethanol/water at $37{ }^{\circ} \mathrm{C}$ observed by Sun et al., ${ }^{43}$ while the $\gamma$-polymorph was observed with indomethacin/PVP solid dispersions in the same study. While studying the dissolution of quench-cooled amorphous indomethacin in water in a flow-through cell at room temperature, Greco and Bogner $^{8}$ detected three polymorphs with confocal Raman microscopy: the $\alpha$ - and $\gamma$-polymorphs as well as an unidentified form with a sharp peak at $1670 \mathrm{~cm}^{-1}$. The form characterized by the peak at $1670 \mathrm{~cm}^{-1}$ has also been observed with Raman spectroscopy mapping at different dissolution time points by Tres et $\mathrm{al}^{33}$ The peak was observed for extrudates of indomethacin with copovidone in a dissolution flow cell at $25{ }^{\circ} \mathrm{C}$ in $\mathrm{pH} 6.8$ phosphate buffer. ${ }^{33}$ While studying aqueous suspensions prepared with amorphous indomethacin at room temperature and a $\mathrm{pH}$ of 6.8 , Surwase et al. ${ }^{23}$ identified indirect conversion of amorphous indomethacin to the $\alpha$ polymorph via the form they denoted as the $\varepsilon$-form (observed after $5 \mathrm{~min}$ ) using ATR-FTIR spectroscopy. At a lower temperature $\left(5{ }^{\circ} \mathrm{C}\right)$ at both $\mathrm{pH} 6.8$ and 1.2 , the $\alpha$-form only became dominant after several sequential transformations involving the $\varepsilon$-, $\zeta$-, and $\eta$-forms.

Table 1. Overview of the Observed Solid-State Forms of Indomethacin before and after 15 min of Dissolution

\begin{tabular}{|c|c|c|c|c|c|c|}
\hline \multirow{3}{*}{ sample } & \multicolumn{6}{|c|}{ solid state forms } \\
\hline & \multicolumn{2}{|c|}{$\mathrm{XRD}$} & \multicolumn{2}{|c|}{ ATR-FTIR $^{a}$} & \multicolumn{2}{|c|}{ CARS/SFG } \\
\hline & before & after & before $^{18}$ & after & before $^{18}$ & after \\
\hline Day 0 & $\mathrm{am}^{b}$ & am, $\varepsilon$ & am & am, $\varepsilon$ (traces) & $\mathrm{am}$ & am, $\varepsilon$ \\
\hline \multicolumn{7}{|c|}{$30^{\circ} \mathrm{C} / 23 \% \mathrm{RH}$} \\
\hline Day 1 & $\mathrm{am}$ & am, $\varepsilon$ & $\mathrm{am}$ & am, $\varepsilon$ (traces) & am, $\gamma$ & am, $\varepsilon, \gamma$ \\
\hline Day 2 & am, $\gamma$ & am, $\gamma$ & am, $\gamma$ & $\gamma$ & am, $\gamma, \alpha$ (traces) & am, $\varepsilon, \gamma$ \\
\hline Day 7 & $\gamma$ & $\gamma$ & $\gamma$ & $\gamma$ & $\gamma, \alpha$ & $\varepsilon, \gamma$ \\
\hline Day 22 & $\gamma$ & $\gamma$ & $\gamma$ & $\gamma$ & $\gamma, \alpha$ (traces) & $\gamma$ \\
\hline \multicolumn{7}{|c|}{$30{ }^{\circ} \mathrm{C} / 75 \% \mathrm{RH}$} \\
\hline Day 1 & am & am & am & am & am, $\alpha$ (traces) & am, $\varepsilon, \gamma, \alpha$ (traces) \\
\hline Day 2 & am, $\gamma$ & am, $\gamma, \varepsilon$ & am, $\gamma$ & $\varepsilon$ & am, $\alpha, \gamma$ (traces) & am, $\varepsilon, \gamma, \alpha$ \\
\hline Day 7 & am, $\gamma, \alpha$ & am, $\gamma, \alpha$ & am, $\gamma$ & am, $\varepsilon, \gamma$ & am, $\alpha, \gamma$ (traces) & am, $\varepsilon, \gamma, \alpha, \eta$ (traces) \\
\hline Day 22 & am, $\gamma, \alpha$ & am, $\gamma, \alpha$ & am, $\alpha$ & am, $\gamma$ & am, $\alpha, \gamma($ traces $)$ & am, $\gamma, \alpha$ \\
\hline
\end{tabular}

${ }^{a}$ Tentative assignments based on IR spectra and PCA. ${ }^{b}$ am $=$ amorphous. 
The results of the present study for day 0 are largely consistent with these studies. However, we observed surface crystallization only to the $\varepsilon$-form of indomethacin, most likely due to the earlier time point of dissolution $(15 \mathrm{~min})$ when solid-state analysis was performed. As confirmed by Surwase et al., ${ }^{23}$ at $\mathrm{pH} 6.8$ and room temperature $\left(25^{\circ} \mathrm{C}\right)$, the conversion of the amorphous to $\alpha$-form can be via the metastable $\varepsilon$ polymorph. If the solid-state analysis in the present study had been performed at a later time point, it is likely that the $\alpha$-form would have been predominantly observed.

The observed $\varepsilon$-form was prepared in pure form and further characterized in this study. Surwase et al. ${ }^{23}$ and Koranne et $\mathrm{al}^{34}$ reported the IR spectra of the $\varepsilon$-polymorph. We believe that the Raman peak at around $1670 \mathrm{~cm}^{-1}$ observed in earlier dissolution studies with amorphous indomethacin ${ }^{8,33}$ was also due to formation of the $\varepsilon$-form. In the present study, this polymorph was confirmed by ATR-FTIR and further characterized by XRD, Raman, CARS, and SFG.

The storage time affected the crystallization and dissolution behavior at both studied storage conditions. The influence of crystallization to the $\gamma$-polymorph during storage at $25{ }^{\circ} \mathrm{C} / 0 \%$ $\mathrm{RH}$ on dissolution was studied by Greco and Bogner. ${ }^{8}$ The dissolution in water of amorphous indomethacin prepared by quench-cooling the melt and stored for 1 week resulted in the presence of three forms identified by confocal Raman microscopy: $\gamma$, the unidentified form with a peak at 1670 $\mathrm{cm}^{-1}$ (which we attribute to the $\varepsilon$-form), and, at the end of experiments, the $\alpha$-form. Samples having a higher percentage of crystallinity stored for 3 weeks showed two forms: $\gamma$ and the form with the Raman peak at $1670 \mathrm{~cm}^{-1}$, while the 6-week sample showed only the presence of the $\gamma$-form. Though the time scales and storage conditions are different, the findings exhibit the same trend as our observations at $30{ }^{\circ} \mathrm{C} / 23 \% \mathrm{RH}$; the 1-day and 2-day samples had similar crystallization behavior to Greco and Bogner's 1-week samples, and the 22day samples corresponded to Greco and Bogner's 6-week sample.

The solid-state results obtained with different analytical techniques are summarized in Table 1. Multiple polymorphs were detected simultaneously with all the used techniques. Of all the analytical techniques used, the combination of CARS and SFG proved to be the most potent in detecting especially the $\varepsilon$ - and $\alpha$-forms of indomethacin after dissolution. Furthermore, nonlinear imaging was especially capable of detecting multiple solid-state forms simultaneously including those present only in traces, including the $\eta$-form. For instance, the amorphous form together with four indomethacin polymorphs $(\varepsilon, \gamma, \alpha, \eta)$ were visualized on the surface of a 7-day tablet stored at $30{ }^{\circ} \mathrm{C} / 75 \% \mathrm{RH}$ after dissolution.

Storage at higher humidity yielded a larger number of observed metastable forms upon dissolution. Surface crystallization in the solid state is now relatively well established. ${ }^{18,30,44-46}$ In the current study, surface crystallization to the $\varepsilon$ - and $\eta$-forms was observed only upon dissolution, indicating the process was solution-mediated.

SFG analysis revealed that, as well as the $\alpha$-form, the $\varepsilon$-, $\eta$-, and $\delta$-forms of indomethacin have noncentrosymmetric crystalline structures, based on their SFG activity. As the crystal structures of the $\varepsilon$-, $\eta$-, and $\delta$-forms have not yet been solved, this provides at least some indication of their possible space groups. In addition to offering such solid-state structure information, multimodal nonlinear imaging involving both CARS and SFG provided greater confidence in resolving these polymorphs. SHG imaging has been used independently in stability and dissolution testing of amorphous drugs and their formulations $^{17,47}$ and was shown to have a large detection range, with a detection limit well-below that for routinely used methods such as DSC or XRPD. These studies however involved the crystallization of the amorphous form into one noncentrosymmetric (SHG-/SFG-active) crystalline form. Here, the SFG analysis is further enriched with vibrational spectroscopic characterization provided by CARS, which enabled identification of different crystal forms of indomethacin that could not have been resolved using SFG only.

The existence of different solid-state forms simultaneously makes some time points more difficult to interpret with the employed nonspatially resolved techniques of XRD and ATRFTIR (combined with PCA). The XRD diffractograms and ATR-FTIR spectra (together with PCA scores) contain contributions from all present solid-state forms. The highly spatially resolved pixel-by-pixel nature of multimodal nonlinear imaging resulted in generally solid-state resolved signals, and minority polymorphs present were efficiently detected, in addition to the main polymorphic forms observed by XRD and ATR-FTIR.

The surface sensitivity of nonlinear optical imaging, combined with noncontact sampling, was important as highlighted by the samples stored at $30{ }^{\circ} \mathrm{C} / 75 \% \mathrm{RH}$ that exhibited dissolution-induced surface formation of the $\varepsilon$-form according to the nonlinear optical imaging results. This signal was not always apparent with XRD or ATR-FTIR spectroscopy. It is likely that the surface layer of these crystals could be so thin that the amorphous indomethacin signal from within the tablet overwhelmed the XRD signal, while the compression on the ATR-FTIR crystal may have been sufficient to induce disordering of the very thin layer of the highly metastable $\varepsilon$ form present.

The observed solid-state results postdissolution can also be compared with those in a study by Priemel at al., ${ }^{32}$ where, after $45 \mathrm{~min}$ of dissolution in stimulated intestinal fluid without enzymes at $\mathrm{pH} 6.8$ and temperature of $37^{\circ} \mathrm{C}$, the presence of the $\alpha$ - and $\gamma$-polymorphs was confirmed with ATR-FTIR, for both initially pure amorphous powders and samples stored for 5 days at the same storage conditions as in the present study $\left(30{ }^{\circ} \mathrm{C} / 23 \% \mathrm{RH}\right)$. It is possible that since their solid-state analysis was performed at a later stage ( $45 \mathrm{~min}$ as opposed to $15 \mathrm{~min}$ in the present study), only the final prevailing polymorphs were present. However, as mentioned above, our results suggest that nonlinear imaging was more sensitive than the ATR-FTIR and could allow detection of the $\varepsilon$-form when ATR-FTIR or XRD may not.

The multimodal nonlinear imaging also provided other solid-state-specific information on distribution at the tablet surface that could not have been derived from the other characterization techniques used. Tablet cracks observed on days 1 and 2 with samples stored at $30{ }^{\circ} \mathrm{C} / 23 \% \mathrm{RH}$ upon dissolution typically contained crystals, as indicated by a SFG signal from these areas. Such surface irregularities or imperfections are typically places where the dissolution process is more rapid, which is why those areas are often referred to as "high energy spots". Interestingly, the "crack-specific" crystallization was much less apparent in the samples stored at 30 ${ }^{\circ} \mathrm{C} / 75 \% \mathrm{RH}$ and may be due to a greater degree of relaxation in these regions when stored at the higher humidity.

The sample presentation during storage also appears to affect dissolution behavior. Priemel at el. ${ }^{32}$ reported that the 
dissolution behavior of amorphous indomethacin particles stored at $30{ }^{\circ} \mathrm{C} / 23 \% \mathrm{RH}$ for 5 days was the same as that of the reference $\gamma$-form. Their study involved intrinsic dissolution experiments performed in simulated intestinal fluid without enzymes at $\mathrm{pH} 6.8$ and with $0.05 \%$ of Tween 20 . Their dissolution profiles corresponded to that of the $\gamma$-form already after 5 days, whereas in the present study at the same storage conditions, the dissolution profiles after 7 days approached that of the $\gamma$-form but were essentially the same as the $\gamma$-form only after 22 days of storage. In their study, individual particles were stored unlike the compressed tablets in this study. The greater overall surface area exposed to the environment during the storage in the study by Priemel et al. ${ }^{32}$ may have affected the subsequently prepared tablet surface crystallinity prior to dissolution. This hypothesis could be tested with nonlinear imaging in the future.

Finally, it is interesting to note that in most of the samples, and in all those stored at higher humidity, multiple solid-state forms were simultaneously observed postdissolution, including two to four different polymorphs. This suggests that the transitions do not necessarily include only sequential transitions, as commonly reported with analytical techniques less efficient at detecting minority species. It is likely that heterogeneous sample characteristics (higher and lower energy amorphous regions on the tablet surfaces induced by particle compression into tablets) and dissolution conditions (different flow dynamics and local solution concentrations induced by surface roughness) contribute to this complex solid-state behavior. It is, however, also possible that the samples are exhibiting probabilistic crystallization behavior, in that more than one solid-state crystalline form may simultaneously crystallize in a single set of conditions.

\section{CONCLUSIONS}

Simultaneous CARS and SFG imaging has been performed to elucidate complex solvent-mediated phase transformations on amorphous tablet surfaces upon dissolution as a function of storage time. SEM, XRD, and ATR-FTIR were used as complementary methods. Overall, multimodal nonlinear imaging proved to be more informative and sensitive than the other techniques used, with distributions of indomethacin polymorphs and the amorphous form efficiently imaged. In particular, the multimodal nonlinear imaging was better able to simultaneously detect multiple polymorphic forms, including those present in trace amounts. SFG also revealed which of the indomethacin polymorphs have noncentrosymmetric structures.

In particular, the recently reported $\varepsilon$-form of indomethacin was observed to form only upon exposure of the amorphous form to the dissolution media, in the early stages of storage at both storage conditions. This form was further characterized with XRD, Raman, and CARS and confirmed to have a noncentrosymmetric structure according to SFG.

It has earlier been established that surface crystallinity is generally more important than overall crystallinity when considering the relationship between crystallinity and the critical quality attribute, dissolution. Thus, multimodal nonlinear optical imaging is a suitable technique for understanding this relationship, especially when developing amorphous drugs and dosage forms. In this present study, very early stage crystallization observed with nonlinear imaging, but not detectable with the conventional solid-state analysis methods, did not have a significant effect on dissolution behavior.
However, trace levels of crystallinity may be important in other samples, and multimodal nonlinear optical imaging combined with dissolution testing provides a means investigating such relationships.

\section{ASSOCIATED CONTENT}

\section{S Supporting Information}

The Supporting Information is available free of charge on the ACS Publications website at DOI: 10.1021/acs.molpharmaceut.8b00840.

ATR-FTIR spectra of tablet surfaces after dissolution and additional CARS and SFG overlay images of tablets stored for 1 and 7 days at $30^{\circ} \mathrm{C} / 75 \% \mathrm{RH}$ (PDF)

\section{AUTHOR INFORMATION}

\section{Corresponding Author}

*E-mail: dunja.novakovic@helsinki.fi.

ORCID 8

Dunja Novakovic: 0000-0003-2058-2194

Clare J. Strachan: 0000-0003-3134-8918

\section{Author Contributions}

The manuscript was written through contributions of all authors. All authors have given approval to the final version of the manuscript.

\section{Notes}

The authors declare no competing financial interest.

\section{ACKNOWLEDGMENTS}

Heikki Räikkönen from the University of Helsinki is gratefully acknowledged for his assistance with the XRD measurements. Marta Cristos from the The Complutense University of Madrid is acknowledged for her help in the initial intrinsic dissolution measurements and method setup. Jaana Koskela from the University of Helsinki is acknowledged for her help in preparing the $\delta$-form of indomethacin. DN gratefully acknowledges the Doctoral Program in Drug Research funding provided by the University of Helsinki. CS acknowledges Business Finland, the Finnish Funding Agency for Innovation (project no. 1245/31/2015), the Academy of Finland (grant no. 289398), and the University of Helsinki for a three-year research project grant (2014-2016).

\section{ABBREVIATIONS}

CARS, coherent anti-Stokes Raman scattering; SFG, sum frequency generation; SHG, second-harmonic generation; SEM, scanning electron microscopy; XRD, X-ray diffraction; PLM, polarized light microscopy; ATR-FTIR, attenuated total reflectance Fourier transform infrared spectroscopy; DSC, differential scanning calorimetry

\section{REFERENCES}

(1) European Directorate for the Quality of Medicines \& HealthCare. European Pharmacopoeia 9.5. http://online6.edqm.eu/ ep $905 /$.

(2) Hancock, B. C.; Parks, M. What is the true solubility advantage for amorphous pharmaceuticals? Pharm. Res. 2000, 17 (4), 397-404.

(3) Murdande, S. B.; Pikal, M. J.; Shanker, R. M.; Bogner, R. H. Solubility advantage of amorphous pharmaceuticals: I. A thermodynamic analysis. J. Pharm. Sci. 2010, 99 (3), 1254-1264.

(4) Debnath, S.; Predecki, P.; Suryanarayanan, R. Use of glancing angle $\mathrm{x}$-ray powder diffractometry to depth-profile phase trans- 
formations during dissolution of indomethacin and theophylline tablets. Pharm. Res. 2004, 21 (1), 149-159.

(5) Ewing, A. V.; Clarke, G. S.; Kazarian, S. G. Stability of indomethacin with relevance to the release from amorphous solid dispersions studied with ATR-FTIR spectroscopic imaging. Eur. J. Pharm. Sci. 2014, 60, 64-71.

(6) Savolainen, M.; Kogermann, K.; Heinz, A.; Aaltonen, J.; Peltonen, L.; Strachan, C.; Yliruusi, J. Better understanding of dissolution behaviour of amorphous drugs by in situ solid-state analysis using Raman spectroscopy. Eur. J. Pharm. Biopharm. 2009, 71 (1), 71-79.

(7) Aaltonen, J.; Heinänen, P.; Peltonen, L.; Kortejärvi, H.; Tanninen, V. P.; Christiansen, L.; Hirvonen, J.; Yliruusi, J.; Rantanen, J. In situ measurement of solvent-mediated phase transformations during dissolution testing. J. Pharm. Sci. 2006, 95 (12), 2730-2737.

(8) Greco, K.; Bogner, R. Crystallization of amorphous indomethacin during dissolution: Effect of processing and annealing. Mol. Pharmaceutics 2010, 7 (5), 1406-1418.

(9) Schmitt, P. D. Recent advances in nonlinear optical analyses of pharmaceutical materials in the solid state. Mol. Pharmaceutics 2017, 14 (3), 555-565.

(10) Windbergs, M.; Jurna, M.; Offerhaus, H. L.; Herek, J. L.; Kleinebudde, P.; Strachan, C. J. Chemical imaging of oral solid dosage forms and changes upon dissolution using coherent anti-Stokes Raman scattering microscopy. Anal. Chem. 2009, 81 (6), 2085-2091.

(11) Jurna, M.; Windbergs, M.; Strachan, C. J.; Hartsuiker, L.; Otto, C.; Kleinebudde, P.; Herek, J. L.; Offerhaus, H. L. Coherent antiStokes Raman scattering microscopy to monitor drug dissolution in different oral pharmaceutical tablets. J. Innovative Opt. Health Sci. 2009, 2 (1), 37-43.

(12) Fussell, A.; Garbacik, E.; Offerhaus, H.; Kleinebudde, P.; Strachan, C. In situ dissolution analysis using coherent anti-Stokes Raman scattering (CARS) and hyperspectral CARS microscopy. Eur. J. Pharm. Biopharm. 2013, 85 (3, Part B), 1141-1147.

(13) Kestur, U. S.; Wanapun, D.; Toth, S. J.; Wegiel, L. A.; Simpson, G. J.; Taylor, L. S. Nonlinear optical imaging for sensitive detection of crystals in bulk amorphous powders. J. Pharm. Sci. 2012, 101 (11), 4201-4213.

(14) Wanapun, D.; Kestur, U. S.; Kissick, D. J.; Simpson, G. J.; Taylor, L. S. Selective detection and quantitation of organic molecule crystallization by second harmonic generation microscopy. Anal. Chem. 2010, 82 (13), 5425-5432.

(15) Schmitt, P. D.; Trasi, N. S.; Taylor, L. S.; Simpson, G. J. Finding the needle in the haystack: Characterization of trace crystallinity in a commercial formulation of paclitaxel protein-bound particles by Raman spectroscopy enabled by second harmonic generation microscopy. Mol. Pharmaceutics 2015, 12 (7), 2378-2383.

(16) Mah, P. T.; Novakovic, D.; Saarinen, J.; Van Landeghem, S.; Peltonen, L.; Laaksonen, T.; Isomäki, A.; Strachan, C. J. Elucidation of compression-induced surface crystallization in amorphous tablets using sum frequency generation (SFG) microscopy. Pharm. Res. 2017, 34 (5), 957-970.

(17) Song, Z.; Sarkar, S.; Vogt, A. D.; Danzer, G. D.; Smith, C. J.; Gualtieri, E. J.; Simpson, G. J. Kinetic modeling of accelerated stability testing enabled by second harmonic generation microscopy. Anal. Chem. 2018, 90 (7), 4406-4413.

(18) Novakovic, D.; Saarinen, J.; Rojalin, T.; Antikainen, O.; FraserMiller, S. J.; Laaksonen, T.; Peltonen, L.; Isomäki, A.; Strachan, C. J. Multimodal nonlinear optical imaging for sensitive detection of multiple pharmaceutical solid-state forms and surface transformations. Anal. Chem. 2017, 89 (21), 11460-11467.

(19) Yamamoto, H. 1-Acyl-indoles. II. A new syntheses of 1-(pchlorobenzoyl)-5-methoxy-3-indolylacetic acid and its polymorphism. Chem. Pharm. Bull. 1968, 16 (1), 17-19.

(20) Borka, L. The Polymorphism of indomethacin. New modifications, their melting behavior and solubility. Acta Pharm. Suec. 1974, 11 (3), 295-303.
(21) Lin, S. Y. Isolation and solid-state characteristics of a new crystal form of indomethacin. J. Pharm. Sci. 1992, 81 (6), 572-576.

(22) Crowley, K. J.; Zografi, G. Cryogenic grinding of indomethacin polymorphs and solvates: Assessment of amorphous phase formation and amorphous phase physical stability. J. Pharm. Sci. 2002, 91 (2), 492-507.

(23) Surwase, S. A.; Boetker, J. P.; Saville, D.; Boyd, B. J.; Gordon, K. C.; Peltonen, L.; Strachan, C. J. Indomethacin: New polymorphs of an old drug. Mol. Pharmaceutics 2013, 10 (12), 4472-4480.

(24) Van Duong, T.; Ludeker, D.; Van Bockstal, P. J.; De Beer, T.; Van Humbeeck, J.; Van den Mooter, G. Polymorphism of indomethacin in semicrystalline dispersions: Formation, transformation, and segregation. Mol. Pharmaceutics 2018, 15 (3), 1037-1051.

(25) Kaneniwa, N.; Otsuka, M.; Hayashi, T. Physicochemical characterization of indomethacin polymorphs and the transformation kinetics in ethanol. Chem. Pharm. Bull. 1985, 33 (8), 3447-3455.

(26) Karmwar, P.; Graeser, K.; Gordon, K. C.; Strachan, C. J.; Rades, T. Effect of different preparation methods on the dissolution behaviour of amorphous indomethacin. Eur. J. Pharm. Biopharm. 2012, 80 (2), 459-464.

(27) Alonzo, D. E.; Zhang, G. G. Z.; Zhou, D.; Gao, Y.; Taylor, L. S. Understanding the behavior of amorphous pharmaceutical systems during dissolution. Pharm. Res. 2010, 27 (4), 608-618.

(28) Mah, P. T.; Peltonen, L.; Novakovic, D.; Rades, T.; Strachan, C. J.; Laaksonen, $\mathrm{T}$. The effect of surfactants on the dissolution behavior of amorphous formulations. Eur. J. Pharm. Biopharm. 2016, 103, 1322.

(29) Karmwar, P.; Graeser, K.; Gordon, K. C.; Strachan, C. J.; Rades, $\mathrm{T}$. Investigation of properties and recrystallisation behaviour of amorphous indomethacin samples prepared by different methods. Int. J. Pharm. 2011, 417 (1-2), 94-100.

(30) Wu, T.; Yu, L. Surface crystallization of indomethacin below Tg. Pharm. Res. 2006, 23 (10), 2350-2355.

(31) Andronis, V.; Yoshioka, M.; Zografi, G. Effects of sorbed water on the crystallization of indomethacin from the amorphous state. J. Pharm. Sci. 1997, 86 (3), 346-351.

(32) Priemel, P. A.; Grohganz, H.; Gordon, K. C.; Rades, T.; Strachan, C. J. The impact of surface- and nano-crystallisation on the detected amorphous content and the dissolution behaviour of amorphous indomethacin. Eur. J. Pharm. Biopharm. 2012, 82 (1), 187-193.

(33) Tres, F.; Treacher, K.; Booth, J.; Hughes, L. P.; Wren, S. A. C.; Aylott, J. W.; Burley, J. C. Indomethacin-Kollidon VA64 extrudates: A mechanistic study of $\mathrm{pH}$-dependent controlled release. Mol. Pharmaceutics 2016, 13 (3), 1166-1175.

(34) Koranne, S.; Thakral, S.; Suryanarayanan, R. Effect of formulation and process parameters on the disproportionation of indomethacin sodium in buffered lyophilized formulations. Pharm. Res. 2018, 35 (11), 214.

(35) Peltonen, L.; Liljeroth, P.; Heikkilä, T.; Kontturi, K.; Hirvonen, J. Dissolution testing of acetylsalicylic acid by a channel flow method-correlation to USP basket and intrinsic dissolution methods. Eur. J. Pharm. Sci. 2003, 19 (5), 395-401.

(36) Sarnes, A.; Østergaard, J.; Jensen, S. S.; Aaltonen, J.; Rantanen, J.; Hirvonen, J.; Peltonen, L. Dissolution study of nanocrystal powders of a poorly soluble drug by UV imaging and channel flow methods. Eur. J. Pharm. Sci. 2013, 50 (3), 511-519.

(37) Sunesen, V. H.; Pedersen, B. L.; Kristensen, H. G.; Müllertz, A. In vivo in vitro correlations for a poorly soluble drug, danazol, using the flow-through dissolution method with biorelevant dissolution media. Eur. J. Pharm. Sci. 2005, 24 (4), 305-313.

(38) Greco, K.; Bogner, R. Solution-mediated phase transformation: Significance during dissolution and implications for bioavailability. J. Pharm. Sci. 2012, 101 (9), 2996-3018.

(39) Slavin, P. A.; Sheen, D. B.; Shepherd, E. E. A.; Sherwood, J. N.; Feeder, N.; Docherty, R.; Milojevic, S. Morphological evaluation of the $\gamma$-polymorph of indomethacin. J. Cryst. Growth 2002, 237-239, $300-305$. 
(40) Hartshorn, C. M.; Lee, Y. J.; Camp, C. H.; Liu, Z.; Heddleston, J.; Canfield, N.; Rhodes, T. A.; Hight Walker, A. R.; Marsac, P. J.; Cicerone, M. T. Multicomponent chemical imaging of pharmaceutical solid dosage forms with broadband CARS microscopy. Anal. Chem. 2013, 85 (17), 8102-8111.

(41) Taylor, L. S.; Zografi, G. Spectroscopic characterization of interactions between PVP and indomethacin in amorphous molecular dispersions. Pharm. Res. 1997, 14 (12), 1691-1698.

(42) Trasi, N. S.; Purohit, H. S.; Taylor, L. S. Evaluation of the crystallization tendency of commercially available amorphous tacrolimus formulations exposed to different stress conditions. Pharm. Res. 2017, 34 (10), 2142-2155.

(43) Sun, D. D.; Lee, P. I. Evolution of supersaturation of amorphous pharmaceuticals: The effect of rate of supersaturation generation. Mol. Pharmaceutics 2013, 10 (11), 4330-4346.

(44) Zhu, L.; Wong, L.; Yu, L. Surface-enhanced crystallization of amorphous nifedipine. Mol. Pharmaceutics 2008, 5 (6), 921-926.

(45) Hasebe, M.; Musumeci, D.; Yu, L. Fast surface crystallization of molecular glasses: creation of depletion zones by surface diffusion and crystallization flux. J. Phys. Chem. B 2015, 119 (7), 3304-3311.

(46) Zhu, L.; Jona, J.; Nagapudi, K.; Wu, T. Fast surface crystallization of amorphous griseofulvin below Tg. Pharm. Res. 2010, 27 (8), 1558-1567.

(47) Elkhabaz, A.; Sarkar, S.; Dinh, J. K.; Simpson, G. J.; Taylor, L. S. Variation in supersaturation and phase behavior of ezetimibe amorphous solid dispersions upon dissolution in different biorelevant media. Mol. Pharmaceutics 2018, 15 (1), 193-206. 